\title{
EFFECT OF DIFLUNISAL ON PLATELET FUNCTION AND BLOOD COAGULATION
}

\author{
C.Th. SMIT SIBINGA \\ Groningen, The Netherlands
}

1 The effect of diflunisal on platelet function and blood coagulation in volunteers was studied. The drug was double-blind tested in 20 healthy males randomly assigned to diflunisal and placebo groups. The effect of high dosage was tested in an open design in six healthy males.

2 Test systems were haemoglobin, $\mathrm{mH}$, blood glucose, platelet counts, bleeding time, ADP- and collagen-induced platelet aggregation, PT, PTT, thrombin time, fibrinogen, antithrombin III, clot lysis, serum FDP and diflunisal blood levels. Paired $t$ and covariance tests were computed.

3 The study design in both the double-blind and the open study was as follows: Acute single dose trial - diflunisal or placebo orally on day 1 . Tests were monitored before and at regular intervals after administration on day 1 and further in the fasting state on days, 2, 4, 8, 10 and 12 . Chronic multiple dose trial - diflunisal or placebo twice daily orally from day 15 to day 21 . The same tests were monitored before and at regular intervals after administration on day 15 , and in the fasting state on days $16,18,21,25,29,31$ and 33 .

4 Diflunisal at the dosages studied did not cause significant changes in any parameter measured at any time. Blood levels of diflunisal were comparable to those obtained during analgesic therapy. In the high dosage open study there were slight effects on platelet aggregation. Bleeding times were not influenced.

\section{Introduction}

The use of antipyretic analgesic drugs is widespread in a large number of clinical conditions. Their use is, however, limited by a number of side-effects such as allergic reactions, effects on organs such as the kidney, and on bone marrow, and the induction of a haemorrhagic diathesis by effects on blood coagulation and platelet function.

Salicylic acid derivatives, and particularly the acetylated compounds, are well known for their effect on platelet number and function (O'Brien, 1968; Zucker \& Peterson, 1970). This effect is of clinical importance, because of the risk of more or less severe haemorrhages during therapy with these substances. There is therefore a strong need for potent analgesic drugs without any effect on haemostasis.

The purpose of the study was to determine the presence, extent and duration of any effect that a single dose or chronic administration of diflunisal may exert on platelet function, fibrin formation, fibrinolysis and fasting blood glucose.

\section{Methods}

Diflunisal was double-blind tested in 20 healthy male volunteers according to the conditions of the Helsinki declaration. In an open study the effect of high dosage was tested in six healthy male volunteers. Test systems included: $\mathrm{Hb}, \mathrm{mH}$, fasting blood glucose, platelet count, bleeding time (standardized Borgghrevink technique), ADP- and collagen-induced aggregation (according to Born), and clot retraction. Fibrin formation was tested with a PT, PTT and thrombin time. Fibrinogen, antithrombin III, 24-h clot lysis and serum FDP according to Merskey, were measured, Diflunisal blood levels were concurrently studied. For statistical analysis means, standard deviations and standard errors of the mean were calculated. Paired $t$ tests for analysis within a group and covariance for relations between the two groups were used. After a drug-free period of two weeks, the volunteers were randomly assigned to placebo or diflunisal groups.

\section{Double-blind design}

Acute single dose trial Volunteers received diflunisal $250 \mathrm{mg}$ or placebo orally on day 1 . Tests for blood coagulation and platelet function were monitored before, 4 and $8 \mathrm{~h}$ after administration, and in a fasting state on days $2,4,8,10$ and 12 .

Chronic multiple dose trial Volunteers received diflunisal $250 \mathrm{mg}$ or placebo twice daily from day 15 to day 21 . The same tests were monitored before, 4 
and $8 \mathrm{~h}$ after administration on day 15 , and in a fasting state on day $16,18,21,25,29,31$ and 33 .

\section{Open high dosage design}

Acute single dose trial Volunteers received diflunisal $500 \mathrm{mg}$ on day 1 . Tests for blood coagulation and platelet function were monitored before, $6 \mathrm{~h}$ after administration on day 1 , and in a fasting state on days 2 and 5.

Chronic multiple dose trial Volunteers received diflunisal $500 \mathrm{mg}$ twice daily from day 8 to day 15 . The same tests were monitored before and $6 \mathrm{~h}$ after administration on day 8 , and in a fasting state on days $9,12,15$ and 19.

\section{Results}

Age (19-36 yr) and weight ranges $(62-87 \mathrm{~kg})$ of the volunteers in all groups were equal. All plain samples for drug analysis in both the study designs taken before treatment exhibited normal background fluorescence, indicating the absence of any salicylates.

In the double-blind study individual diflunisal blood levels increased to $35.9 \mu \mathrm{g} / \mathrm{ml}$ (mean 24 ; s.d. \pm 6.8 ) after a single dose of diflunisal $250 \mathrm{mg}$. In the chronic multiple dose experiment an individual peak of $60.0 \mu \mathrm{g} / \mathrm{ml}$ (mean 37.9; s.d. \pm 9.1 ) was reached after 1 week on diflunisal $250 \mathrm{mg}$ twice daily. On day 4 in the single dose and day 25 in the multiple dose experiment, the diflunisal blood levels had decreased to background levels. The blood levels reached were comparable to those obtained during analgesic therapy using the same dosage scheme of diflunisal $250 \mathrm{mg}$ twice daily. In the high dosage study individual diflunisal blood levels increased to $48.8 \mu \mathrm{g} / \mathrm{ml}$ (mean 45.3 ; s.d. \pm 4.8 ) after a single dose of diflunisal $500 \mathrm{mg}$. In the chronic multiple dose experiment a level of $110.5 \mu \mathrm{g} / \mathrm{ml}$ (mean 84.4; s.d. \pm 13.9) was reached after 1 week on diflunisal $500 \mathrm{mg}$ twice daily. On day 5 in the single dose and day 19 in the multiple dose experiment the diflunisal blood levels had decreased to background levels.

\section{References}

BORN, G.V.R. (1962). Aggregation of blood platelets by adenosine diphosphate and its reversal. Nature, 194, 927-929.

O'BRIEN, J.R. (1968). Effect of anti-inflammatory agents on platelets. Lancet, i, 894-895.

TEMPERO, K.F., STEELMAN, S.L., BESSELAAR, G.H., SMIT SIBINGA, C.Th., de SCHEPPER, P.J.,
Fasting blood glucose levels showed no differences between the groups in both studies. In the double-blind study, platelet function showed no significant change compared with the placebo group either in the acute or in the chronic trial (and also compared with the starting values within the diflunisal group). Of particular interest is the absence of any effect at any time on bleeding time, ADP-induced platelet aggregation (measured by the slope), $A_{\text {max }}$, the disaggregation phenomenon and the collagen-induced platelet aggregation (measured by the latency time), the slope, the initial $A_{\max }$, and the presence of a secondary release wave in the diflunisal group, compared with the control group.

In the high dosage study, again no effect was registered at any time on bleeding time. ADP-induced platelet aggregation showed no effect on the $A_{\text {max }}$, the slope and the disaggregation phenomenon in the single dose trial, whereas only slight effects were measured on the slope and the disaggregation phenomenon in the multiple dose trial. Here, the slope showed a tendency to flattening and a slight but consistent disaggregation phenomenon occurred.

None of the other parameters of blood coagulation or platelet numbers showed any significant change at any time during the whole experiment. No adverse laboratory or clinical experiences were noted.

\section{Conclusions}

Diflunisal, which has already been shown to have a potential antipyretic and analgesic effect (Tempero et al., 1975), seems to have no measurable effect on platelet function nor on platelet count, on fasting blood glucose, on fibrin formation or fibrinolysis (as tested in a double-blind design in 20 healthy male volunteers). Only at a dose of $500 \mathrm{mg}$ twice daily for 1 week was a slight effect on platelet aggregation observed, although bleeding times were not influenced at all. We consider, therefore, that diflunisal is of special interest for analgesic therapy in patients with a potential or manifest bleeding tendency.

I thank Dr G.O. Breault, Dr P.J. de Schepper and Dr K.F. Tempero for contributions.

TJANDRAMAGA, T.B., DRESSE, A. \& GRIBNAU, F.W.J. (1975). Special studies on diflunisal a novel salicylate. Clin. Res., 23, 224A.

ZUCKER, M.B. \& PETERSON, J. (1970). Effect of acetylsalicylic acid, other nonsteroidal anti-inflammatory agents, and dipyridamole on human blood platelets. $J$. Lab. clin. Med., 76, 66-75. 